\title{
Moral Reasoning and Decisions in Dilemmas of Neonatal Care
}

\author{
DANIEL CANDEE, ${ }^{(10)}$ T. JOSEPH SHEEHAN, CHARLES D. COOK, SUSAN D. R. HUSTED, AND \\ MARK BARGEN \\ Department of Research in Health Education, University of Connecticut Health Center, Farmington, Connecticut \\ [D.C., T.J.S., S.D.R.H.J; Department of Pediatrics, Downstate University Medical Center, \\ Brooklyn New York [C.D.C.; and Sperry-Univac, 12900 Avenue of the Americas, New York, New York [M.B.], USA
}

\section{Summary}

The relationship between levels of moral reasoning and decisions in dilemmas of neonatal care was investigated in a sample of $\mathbf{4 5 2}$ pediatricians. Subjects included residents, faculty members, and practitioners recruited from a variety of university-affiliated and community hospitals.

It was hypothesized that physicians whose moral reasoning was more fully developed would less actively treat particular cases. Such cases might include those where a patient's family requested such a limit (designated "negative family attitude") or the quality of life likely to be led after therapy was so low as to preclude even a minimal degree of human activity or social interaction (designated "unsalvageable prognosis").

The hypothesis was tested through the use of two questionnaires. The first questionnaire, devised by Crane, assessed the physician's reported degree of activism in treating six cases of infants born with severe defects. The structure of moral reasoning was measured by a second questionnaire, Rest's Defining Issue Test. Subjects were scored by the degree to which they use universal, ethical principles in resolving a series of moral dilemmas.

Results of the absolute level of activism (Table 1) showed that among both residents and postresidents, the degree to which cases are actively treated depends, for salvageable patients, on the type of damage and on the possibility for research. Results involving moral reasoning showed a different pattern among residents and postresidents. Among residents, a significant correlation exists between principled reasoning and the absence of active treatment $(r=-0.41$, Form A; $r=-0.23$, Form B). As predicted, such correlations were strongest for cases of negative family attitude or of unsalvageable prognosis. The pattern of correlations among postresidents showed either no relationship to moral reasoning or the reverse of the residency pattern $(r=-0.08$, Form $A ; r=0.30$, Form B).

The influence of the type of institution a resident operates within was assessed by analysis of variance. Inasmuch as moral reasoning and institutional type both had significant main effects (Form A), their magnitude differed. Institutional type accounted for $43 \%$ of the variation in mean activism scores whereas moral reasoning accounted for only $4 \%$; however, because one could, a priori, expect institutional norms and customs to be powerful determinants of behavior, any additional, identifiable influence deserves attention. The structure of individuals' moral reasoning seems to be such an influence.

\section{MORAL REASONING AND DECISIONS IN DILEMMAS OF NEONATAL CARE}

Among the most important decisions a pediatrician makes is the type of treatment he or she will employ in cases of children born with severe defects. Such decisions lie at the intersection of both medical and moral concerns. Questions of whether to provide heroic care, care that is often expensive, painful, and unfortunately often futile, are not simply matters of technical expertise. They involve value choices.

How does a physician make decisions on such matters? What factors are most influential in his or her thinking? Answers to some of these questions were provided by Crane (1) who surveyed 922 practicing physicians and residents in pediatrics and in medicine, concerning their attitudes towards treatment of the critically ill. Crane found that the degree of activism advocated by such physicians differed greatly depending on such case factors as the possibility for recovery, the type of damage sustained, and the attitude of the patient or family towards aggressive medical treatment, and on such physician factors as professional status prestige of hospital affiliation and religious background. Further, she found a significant relationship between the advocacy of attitudes concerning the degree of activism and the actual practice of it. Although such findings clarify the considerations likely to be involved in a physician's decision, they do not tell us whether these considerations are part of any larger psychologic structures.

The possibility that such elements stem from an individual's general structure of moral reasoning is suggested by a growing tradition of research in that field. Work done by Kohlberg $(2,3)$ has shown that the underlying principles or "cognitive structures," which an individual uses to resolve moral dilemmas, develops through a series of six sequentially ordered stages. The stages begin with a punishment and obedience orientation (Stage 1) and develop through a conventional acceptance of societal or in-group rules (Stages 3 and 4), to a postconventional autonomy in which moral decisions are made on the basis of universally valid, ethical principles (Stages 5 and 6). At each succeeding state, there is a greater appreciation of the welfare of others and a greater desire to resolve moral dilemmas in a fair and equitable manner.

When applied to the realm of decision-making in cases of children born with severe defects, the implication of this research is that higher stage reasoning, because it is based on a greater respect for personal dignity and individual rights, will lead physicians holding such reasoning to be more sensitive to characteristics of the patient and of the situation which affects those rights. In order to test this proposition, we conducted a survey of 452 pediatric residents, faculty members, and practitioners in which we measured the relationship between moral reasoning; and attitudes towards the treatment of these neonates. We hypothesized that physicians whose moral reasoning was more fully developed would be less active in treating particular cases. Such cases might include those where the patient's family requested such a limit (i.e., cases of negative family attitude in Crane's questionnaire) or where the quality of life likely to be led after therapy was so low as to preclude even a minimal degree of human activity or social interaction (cases considered to be "unsalvageable" in Crane's questionnaire). 
Table 1. Mean activism scores for pediatric cases

\begin{tabular}{lccccc}
\hline & \multicolumn{2}{c}{ Residents $^{1}$} & & \multicolumn{2}{c}{ Postresidents $^{2}$} \\
\cline { 2 - 3 } \cline { 5 - 6 } & Mean & S.D. & & Mean & S.D. \\
\hline Salvageable & & & & \\
$\quad$ Physical damage & & & & \\
$\quad$ SES+ (A2) & & & & & \\
SES- (B2) & 1579 & 3.29 & & 15.68 & 3.96 \\
Mental damage & & 4.24 & & 14.56 & 4.07 \\
$\quad$ SES+, family attitude+ (A4) & 16.67 & 3.15 & & 15.64 & 3.91 \\
SES+, family attitude- (A3) & 18.20 & 3.03 & & 17.10 & 3.00 \\
SES-, family attitude+ (B3) & 15.19 & 4.16 & & 12.41 & 4.17 \\
SES-, family attitude- (B4) & 14.50 & 4.50 & & 12.41 & 4.44 \\
$\quad$ Doctor's error (A5) & 19.51 & 1.63 & & 19.84 & 1.79 \\
$\quad$ Mechanical error (B5) & 18.89 & 2.35 & & 18.56 & 1.93 \\
Unsalvageable & & & & \\
Physical damage & & & & \\
$\quad$ Research (B la) & 16.95 & 3.04 & & 16.40 & 3.21 \\
$\quad$ No research (B1b) & 15.63 & 3.85 & & 14.03 & 3.78 \\
Mental damage & & & & \\
$\quad$ Research (Alb) & 15.01 & 4.28 & & 14.51 & 4.50 \\
$\quad$ No research (Ala) & 10.71 & 3.61 & & 8.79 & 2.55 \\
\hline
\end{tabular}

${ }^{1}$ Residents $n=85$ (Form A), $n=100$ (Form B).

${ }^{2}$ Postresidents $n=76$ (Form A), $n=78$ (Form B).

${ }^{3}$ Number in parentheses is case number. Content of cases is as follows: (1) Anencephaly (A1), Hypoplastic ventricle (B1); (2) High lumbar myelomeningocele with no nerve function in legs, bladder, or rectum (A2, B2); (3) Downs Syndrome with severe respiratory distress (A3, B3); (4) Premature separation of the placenta resulting in marked spasticity and hypertonia (A4, B4); and (5) Insufficient clamping of umbilical cord stump by doctor with resulting bleeding (A5). Fourteen-hundred $\mathrm{g}$ infant is exposed to $107^{\circ} \mathrm{F}$ for at least $30 \mathrm{~min}$ due to mechanical failure of incubator with febrility, marked lethargy, lack of reflexes, dehydration, and acidotia resulting (B5).

\section{SUBJECTS}

Between 1974 and 1977, questionnaires measuring both moral reasoning and decisions in dilemmas of neonatal care were distributed either individually or in groups of 620 practicing pediatricians, pediatric faculty members, and residents in pediatrics. The number of subjects who returned completed instruments was 452 or $73 \%$ of the total number of recipients. Nonrespondents were as likely to come from any of the subgroups. Two hundred sixty-six respondents were residents, 186 were postresidents. Postresidents consisted of members of the American Board of Pediatrics and the American Board of Pediatric Research (51), members of several medical school faculties (97), and former house officers at a prestigious university hospital now in private practice (38). One hundred thirty-six of these subjects identified themselves primarily as university faculty members; 50 identified themselves as practitioners. All postresidents were graduates of U.S. medical schools. Their average age was 44 years.

Residents were drawn from two prestigious university hospitals (56), two university hospitals (168), and three community hospitals (42). Designation of a hospital as prestigious was made on the basis of national survey conducted with 28 professors of pediatrics. The designation was given to programs that were perceived by these judges as falling in the top $10 \%$ of pediatric residency programs nationwide. One hundred eleven residents were graduates of U.S. medical schools while 155 were foreign medical school graduates. All foreign graduates were found at either the university or community hospitals. Residents averaged 29 years of age. Three hundred sixty-six subjects were in the main sample. Because questionnaire means can vary from sample to sample, 86 subjects were used to comprise a second, confirmatory sample.

Because the entire sample is neither random nor stratified, results must be interpreted with reference to those subjects actually studied. As a note of comparison, the Journal of the American Medical Association reports that $17.8 \%$ of all pediatricians who had actually been certified as of December 31,1977 were graduates of foreign medical schools $(3,117$ out of 17,453$)(4)$; however, the percentage of foreign doctors in residency at the time of this study probably surpassed this figure because their numbers had been growing, at least through 1977 . Thus, our sample of residents, 58\% of whom were graduates of foreign medical schools, moderately overrepresents the non-U.S. group.

\section{MEASURES}

Attitudes towards treatment. All subjects completed two questionnaires. The first, devised by Crane (1), assessed the physician's reported degree of activism in the treatment of six cases involving infants born with severe defects. The questionnaire systematically varied six factors: prognosis (salvageable, i.e., whether the defect could be corrected to allow a normal degree of human interaction, versus unsalvageable), type of damage (mental defect versus physical defect), family attitude (high, i.e., positive towards active treatment, versus low, i.e., negative towards active treatment), socioeconomic status (SES) of the patient or his family, type of error (whether the patient's defect was due to a doctor's treatment error or to a mechanical error), and the opportunity for research (yes versus no).

In order to provide counterbalanced conditions for each case, two forms of the questionnaire were used. Each subject received one form only. A typical item is Case A2, an infant born with high lumbar myelomeningocele. The case is one of physical damage with a salvageable prognosis. In this situation the factor of family SES was varied. On Form A the family was described as being "well-educated and financially comfortable," (i.e., high SES) whereas on Form B the family was described as "not having finished high school," (i.e., low SES). Other factors such as the family's attitude toward treatment were not specified.

Following each case, there appeared seven choices for action ranging from minimal support (e.g., paint with antibiotic) to heroic treatment (e.g., resuscitation). Respondents were asked to indicate whether they would, maybe would, or would not perform each type of action. To illustrate, Case A2 follows.

An infant is born with high lumbar myelomeningocele. He has no nerve function in his legs and no bladder or rectal control. The parents of the child are well educated and financially comfortable. Which of the following would you be likely to perform? (Check yes, maybe, or no for each item.)

(1) Treat myelomeingocele locally by painting the area with an antibiotic preparation.

(2) Arrange for operation for early closure of defect.

(3) Manage urinary tract infection.

(4) Perform credé masage on the bladder.

(5) Arrange shunt operation if hydrocephalus developed.

(6) If the infant developed meningitis, would you treat him?

(7) If the infant had a cardiac arrest, would you resuscitate him?

Activism indicies. A subject's activism score on each case was determined by awarding three points for each action the respondent indicated he or she would perform, two points for each action marked "maybe," and one point for each action that would not be performed. An individual's story activism score was able to range from seven to 21. An index of average total activism was computed across the 12 stories. Any one subject received scores on only half of these cases, i.e., those appearing on his or her form. A second activism index reflecting intraindividual variability was computed by determining the standard deviation for each subject across all stories completed.

Defining issues test. The Defining Issues Test (DIT) is a structured measure designed by Rest (5) on the basis of Kohlberg's theory of cognitive moral deveopment. Subjects are presented with six moral dilemmas and are asked, for each dilemma, to 
choose from a set of 12 considerations those which the subject believes are most important in resolving the issue at hand. The considerations have been preselected to represent several of Kohlberg's moral stages. An individual' score is expressed in terms of the percentage of responses which have been keyed at the principled stages of moral reasoning (Stages 5 and 6) to which the subject gives high priority.

An example of one dilemma used in the DIT is that of a husband, Heinz, who must decide whether to steal a drug to save his dying wife when all reasonable and legal means to obtain the rare drug have failed. The consideration of whether "it is natural for a loving husband to care so much for his wife" is scored at Stage 3 because it decides moral issues on the basis of maintaining good relationships. The consideration of "what values are going to be the basis for governing how people act towards each other" represents the stage of universal ethical principles (Stages 5 and 6).

Research with over 5,000 subjects reviewed by Rest (5) indicates that the internal consistency reliabiilty of the DIT is 0.77 , and testretest reliability is 0.80 . The measure's stability is also evidenced by the fact that no significant differences appeared between testretest means in three 1 -month samples reported. Construct validity of the DIT is demonstrated by its success in measuring sequential development over time. Results of the two longitudinal studies reported by Rest show increasing P-scores at each later interval of testing. The convergent validity of the DIT is revealed by its correlation with the theoretically predicted variables of comprehension of moral issues, law and order orientation, and political tolerance. The correlation between the DIT and Hohlberg's moral judgment interview in a sample of 45 physicians was found to be $r=0.78(6)$. In short, the DIT seems to measure a stable underlying structure of moral reasoning, not simply a set of varying attitudes.

\section{RESULTS}

The first step in analyzing data is to determine that a phenomenon worthy of interpretation exists. In this study the basic phenomenon was variability of treatment among cases. A repeated measures analysis of variance revealed that there is indeed significant variability in the degree to which cases are treated. Mean activism scores for each of the 12 cases are reported in Table 1. Because the mean activism score for residents (15.41) was significantly higher than for postresidents $(14.51), t=2.39, P<0.05$, we report the results for each group separately.

The cases in Table 1 are identified by their case number (in parentheses) and by those factors that were specified for that case in the questionnaire. To determine the content of the case, see footnote 3. For example, the first case listed in Table 1, Case A2, is a case of myelomeningocele (see table footnote 3 ), which has a salvageable prognosis, is a physical defect, and in which the family is of high socioeconomic status. Other factors, such as the family attitude towards treatment, were not specified. As Table 1 indicates, the highest degree of activism for both residents and postresidents was found on Case A5, insufficient clamping of the umbilical cord. The least activism was found on Case Ala, anencephaly, no research condition.

Although the average level of activism was somewhat higher among residents than among postresidents, the relative order of activity on the cases was almost identical for the two groups (Spearman $\mathrm{R}=0.87$ ). This order is also quite consistent with the results found by Crane in her original study (Spearman $R=0.85$ ) (1).

Because the cases on the Crane questionnaire follow a design structure, they can be combined to reveal larger effects. For example, a variable that measures activism when family attitude is positive can be constructed by averaging the means of Case A4 and B3 (17.60). This variable is significantly higher than is the mean activism obtained when family attitude is negative (Cases $\mathrm{A} 3$ and B4, 14.39).

Other significant effects include, among salvageable cases, type of damage (mental, 16.00 versus physical, 14.90), and type of error (doctor, 19.61 versus machine, 18.71). Among unsalvageable cases, significant effects include type of damage (physical, 16.29 versus mental, 12.86), and researchability (yes, 15.11 versus no, 10.43). The confirmation sample of 86 residents replicated only the family attitude effect among salvageable cases but all effects among unsalvageable cases.

Moral reasoning and attitudes towards treatment. We turn now to the relationship between moral reasoning and pediatricians' attitudes towards treatment of the defective newborn. Mean DIT scores were $27.45($ S.D. $=10.10)$ for residents, $29.70($ S.D. $=8.71)$ for postresidents. Correlations between the DIT and activism appear in Table 2 . As can be seen, the degree of activism averaged across all caes is significantly related to moral reasoning among residents $(r=-0.41$ for Form $\mathrm{A}$ and -0.23 for Form B). The negative sign on the correlation indicates that those residents who scored high in moral reasoning were less active in treating these cases than were residents whose moral reasoning was low. Our first hypothesis, that higher moral reasoning would be related to lesser levels of activism, is confirmed among residents only.

Verification of this hypothesis among residents is also indicated by the significant correlation between moral reasoning and a measure of the variability in response to the six cases (see average variability column Table 2). As shown, higher moral reasoners were more variable in their responses to the cases then were lower moral reasoners. This can be interpreted as demonstrating that higher reasoning residents were more sensitive to characteristics of each situation and varied their treatment accordingly. In comparison, lower reasoning residents seem to treat cases more uniformly.

The relationship between moral reasoning and activism among postresidents, though, is considerably different. For this group, moral reasoning is unrelated to average activism on Form A, and is significantly related in the positive direction on form $\mathrm{B}$. There was no relationship between moral reasoning and the variability of activism on either form for this group.

Our second part of the hypothesis, predicting the particular cases in which a correlation between moral reasoning and lesser levels of activism might be found, can be tested using the data reported in Table 3. An examination of those correlations reveals that, among residents, the lesser degree of activism advocated by higher moral reasoners is, as predicted, almost entirely due to cases that are either unsalvageable (Cases Ala, Alb, Bla, and $\mathrm{B} 1 \mathrm{~b}$ ) or where the family attitude is negative (Cases A3 and B4). Our second hypothesis is also supported, though again, only among residents.

Can the different patterns of relationship between moral reasoning and activism for residents and for postresidents be explained by other variables? The most likely variable for all pediatricians is the type of institution in which one works. For postresidents, factors such as age and type of practice (i.e., faculty or practitioner) may also be relevant. To further explain the observed relationships, we conducted several analyses of variance testing these factors.

Looking first among residents only, we asked whether the activism-moral reasoning correlation could be due to differences in institutional policies. If higher stage residents tend to come from one or two institutions and if the norm at these institutions is to refrain from actively treating certain types of patients, then

Table 2. Pearson correlations between moral reasoning and activism indices

\begin{tabular}{llclcc}
\hline & \multicolumn{2}{c}{ Average activism } & & \multicolumn{2}{c}{ Average variance } \\
\cline { 2 - 3 } \cline { 5 - 6 } & Form A & Form B & & Form A & Form B \\
\hline Residents ${ }^{1}$ & $-0.41^{3}$ & $-0.23^{3}$ & & $0.39^{3}$ & $0.26^{3}$ \\
Postresidents & -0.08 & $0.30^{3}$ & & 0.07 & -0.06 \\
\hline
\end{tabular}

'Residents $n=62$ (Form A), $n=59$ (Form B).

${ }^{2}$ Postresidents $n=85$ (Form A), $n=75$ (Form B).

${ }^{3} P<0.05$. 
Table 3. Pearson correlations between moral reasoning and activism for individual cases

\begin{tabular}{lcc}
\hline & Residents $^{1}$ & Postresidents $^{2}$ \\
\hline Salvageable & & \\
Physical damage & & \\
SES+ (A2) & -0.14 & 0.00 \\
SES- (B2) & -0.35 & $0.27^{3}$ \\
Mental Damage & & \\
SES+, family attitude+ (A4) & $-0.25^{3}$ & 0.00 \\
SES-, family attitude+ (B3) & 0.11 & 0.14 \\
SES+, family attitude- (A3) & $-0.27^{3}$ & -0.15 \\
SES-, family attitude- (B4) & $-0.40^{3}$ & 0.13 \\
Doctor's error (A5) & 0.05 & -0.02 \\
Mechanical error (B5) & 0.07 & $0.23^{3}$ \\
Unsalvageable & & \\
Physical damage & & \\
Research (B1a) & $-0.24^{3}$ & $0.21^{3}$ \\
No research (B1b) & $-0.30^{3}$ & $0.19^{3}$ \\
Mental damage & & \\
Research (A1b) & $-0.34^{3}$ & -0.02 \\
No research (A1a) & $-0.40^{3}$ & 0.01 \\
\hline
\end{tabular}

${ }^{1}$ Residents $n=62$ (Form A), $n=59$ (Form B).

${ }^{2}$ Postresidents $n=85$ (Form A), $n=77$ (Form B).

${ }^{3} P<0.05$.

${ }^{4}$ Number in parentheses is case number. Content of cases is as follows: (1) Anencephaly (A1), hypoplastic ventricle (B1); (2) High lumbar myelomeningocele with no nerve function in legs, bladder, or rectum (A2, B2); (3) Downs Syndrome with severe respiratory distress (A3, B3); (4) Premature separation of the placenta resulting in marked spasticity and hypertonia (A4, B4); and (5) Insufficient clamping of umbilical cord stump by doctor with resulting bleeding (A5). Fourteen-hundred $g$ infant is exposed to $107^{\circ} \mathrm{F}$ for at least $30 \mathrm{~min}$ due to mechanical failure of incubator with febrility, marked lethargy, lack of reflexes, dehydration, and acidosis resulting (B5).

the moral reasoning-activism association may be artificial. The observed correlation may merely reflect the association of both activism and moral reasoning with certain institutional policies. In order to test this proposition, a two-way analysis of variance was conducted. The factors included moral reasoning (high, medium, low) and institutional type (university prestige, university urban, and community). Average activism was the dependent variable.

The purpose of this analysis was to determine whether an interaction between moral reasoning and institutional type exists. If both main effects were found to be significant but not the interaction, then we would be able to speak of the independent influence of both moral reasoning and institutional type on activism. Indeed, for Form A stories, this was the case (Table 4). Although the correlation between moral reasoning and institutional type itself was substantial (i.e., higher stage residents were more prominent at prestigious hospitals, $r=0.50$ ), each factor held a significant amount of variation uniquely in common with activism.

Inasmuch as both moral reasoning and institutional type appear to influence attitudes towards the treatment of the critically ill, the magnitude of their association differs. The column labeled Eta Squared (Table 4) is the percentage of variation in activism scores that can be accounted for by particualr factors. It is calculated by dividing the sum of squares for the relevant factor by the total sum of squares. As can be seen in Table 4, institutional type accounts for a full $43 \%$ of activism variance whereas moral reasoning accounts for only $4 \%$. Although the influence of moral reasoning is significant, i.e., not zero, it is only one-tenth as great as the influence of hospital type. A similar analysis using form B stories reveals that only the institutional type effect was significant (Table 4). The results in Table 4 suggest the strong effect of institutional norms on attitudes towards the treatment of neonatal defects.

Further evidence for the existence of such norms can be seen by including data from postresidents. The analysis of variance with this mixed group focused on the independent variables of institutional variable. Because very few faculty members were low in moral reasoning, the factor could not be included in the analysis. As Table 5 shows, there is considerably more similarity in average activism scores between faculty and residents of a given hospital type than there is between faculty and resdients at one hospital type and fellow faculty or residents at another. This is reflected statistically in a significant institution type effect $(\mathrm{F}=11.53, P<$ 0.001 for Form A; F $=12.39, P<0.001$ for Form B), but no status or interactional effect. Further analyses of the postresidency data alone, such as dividing the group by age or by faculty member versus private practitioner, yielded no differences in average activism.

\section{DISCUSSION}

The major findings of this study fall into three areas: the stability of attitudes, the determinants of attitudes, and the relationship of attitudes to moral reasoning. The high correlations of cases ranked in order of activism in our data with those in Crane's original data and between residents and postresidents in our own study suggest that attitudes towards the treatment of neonatal defects are relatively stable and enduring features of the pediatrician's culture. Such attitudes are determined by factors within the case, within the individual, and within the institution. Among case factors, the largest determinant in salvageable cases is the family's attitude towards treatment, and in unsalvageable cases, the possibility of research. Among individual factors, our results showed that, particularly among residents, the content of a physician's attitude is related to his or her general structure of moral thought. Residents whose moral reasoning was more developed were less aggressive in their treatment of neonatal defects, especially in cases where the family requested such limits or where the quality of life after treatment was likely to preclude even a minimal degree of human or social interaction. Further, they showed greater variability among cases suggesting a more complex or more discriminating approach to treatment.

Table 4. Analysis of variance on average activism by moral reasoning and institutional type $e^{1}$

\begin{tabular}{lrrrrr}
\hline \multicolumn{1}{c}{ Source } & $\begin{array}{c}\text { Sum of } \\
\text { squares }\end{array}$ & df & MS & F & $\begin{array}{c}\text { Eta } \\
\text { squared }\end{array}$ \\
\hline Form A & & & & & \\
$\quad$ Moral reasoning & 14.71 & 2 & 7.35 & $3.14^{2}$ & 0.04 \\
$\quad$ Institutional type & 154.67 & 2 & 77.33 & $33.10^{3}$ & 0.43 \\
$\quad$ Moral X type & 8.97 & 4 & 2.44 & 0.96 & 0.02 \\
Form B & & & & & \\
$\quad$ Moral reasoning & 11.74 & 2 & 5.87 & 1.31 & 0.03 \\
$\quad$ Institutional type & 47.34 & 2 & 23.67 & $5.28^{3}$ & 0.11 \\
$\quad$ Moral X type & 39.72 & 4 & 9.93 & 2.21 & 0.09 \\
\hline
\end{tabular}

${ }^{1} N=70$ (Form A) and $N=74$ (Form B). Subjects were pediatric residents. Total sum of square is 361.38 (Form A) and 428.70 (Form B). Total SS includes explained sources and residual.

${ }^{2} P<0.05$.

${ }^{3} P<0.001$.

Table 5. Average activism scores by status and institutional type $e^{1}$

\begin{tabular}{lcc}
\hline & \multicolumn{2}{c}{ Status } \\
\cline { 2 - 3 } Institution & Faculty & Residents \\
\hline Form A & $14.00^{1}$ & 13.22 \\
$\quad$ University-prestige & 15.95 & 16.94 \\
$\quad$ University-urban & 14.75 & 15.03 \\
Form B & 16.46 & 17.15 \\
$\quad$ University-prestige & \\
University-urban &
\end{tabular}

${ }^{1}$ University-prestige faculty $n=40$; university-prestige residents $n=$ 35; University-urban faculty $n=31$; and Unversity-urban residents $n=$ 23 . 
Why should the relationship between moral reasoning and activism among residents be greatest on cases involving low family attitude or unsalvageable prognosis? The reason may be that these cases allow for the greatest degree of judgment. They are cases in which one factor (either low family attitude or unsalvageability) mitigates against aggressive treatment; thus, a tension is created between the general norm of active treatment and the special norm of limited treatment in this case. Because moral reasoning is a decision-making mechanism, which serves to resolve conflicts between competing norms, it is likely that these situations would be the ones to demonstrate the influence of moral reasoning most clearly. In comparison, cases of salvageable prognosis where family attitude is either positive or unspecified are consensus situations. Activism means for these cases were generally the highest, approaching the maximum degree of active treatment. Because disputes over the degree of treatment in these cases were small, the effect of moral reasoning was not as noticeable.

A third source of influence on treatment attitudes is the type of institution a pediatrician operates within. The existence of clear institutional norms may be seen in our findings in that there is a greater similarity of attitudes among faculty and residents of the same institution than among either faculty along or among residents alone at different institutions. In terms of the magnitude of influence, we found institutional type to be, particularly for residents, the largest source. This does not mean, however, that the influence of individual moral reasoning is unimportant. One could, a priori, expect institutional norms and customs to be the most powerful determinants of behavior. Any additional identifiable influence, even if small in terms of the amount of behavior explained, deserves attention. The predominance of higher stage moral reasoners at university prestige institutions may have affected the moral "atmosphere" of those institutions, thereby enabling faculty and residents to question the uncritical use of heroic action in all cases of severe illness. Perhaps a "critical mass" of principled physicians is required before there can be substantial sentiment in favor of establishing a policy which alters the doctor's traditional role.

An unexpected finding of the study was the different pattern of correlations found between moral reasoning and attitudes among residents and among postresidents. In contrast to residents, there was either no relationship or, in the case of average activism on Form B, a positive relationship between moral reasoning and attitudes among postresident pediatricians. The differences in patterns between residents and postresidents could not be explained by considering the additional factors of numbers of years out of medical school or one's status as a faculty member or a practitioner.
In our opinion, the most cogent explanation for the different pattern lies in the paucity of subjects with very low DIT scores among the postresident group. Whereas $38 \%$ of the pediatric residents had DIT scores below 20 , only $10 \%$ of the postresidents did. One explanation for the greater range of DIT scores among residents is that they are still in the process of constructing their moral philosophies. Because residents are less certain of their moral attitudes, they may choose a wider range of stage responses when presented with dilemmas such as those in the DIT. A second explanation may be that the resident group contained a greater ethnic diversity. Postresidents were nearly all of U.S. origin, whereas many residents were of foreign nationality. On the basis of our study, it appears that a relationship between moral reasoning and lesser levels of activism in the treatment of neonatal defects is most likely to be found in those samples which contain the gretest diversity of moral thought.

Finally, we should remind the reader that we have studied pediatricians' decisions as attitudes, not their decisions in practice. The relationship between the two remains to be uncovered.

\section{REFERENCES AND NOTES}

1. Crane, D.: The sanctity of social life: physicians' treatment of critically ill patient. (Russell Sage Foundation, New York, 1975).

2. Kohlberg, L.: Stage and sequence: the cognitive-developmental approach to socialization. In: D. Goslin: Handbook of Socialization Theory and Research. (Rand-McNally, Chicago, 1969).

3. Kohlberg, L.: The cognitive-developmental approach to moral education. Phi Delta Kappan, 56: 670 (1975).

4. Medical schools in the United States. JAMA, 238: 2828 (1977).

5. Rest, J.: Development and Judging Moral Issues. (University of Minnesota Press, Minneapolis, 1979)

6. Sheehan, T. J., Husted, S. D. R., Candee, D., Cook, C. D., and Bargen, M.: Moral judgment as a predictor of clinical performance. Evaluation and the Health Professions, 3: 393 (1980).

7. This study was conducted under strict adherence to human experimentation guidelines. An elaborate code was developed to assure anonymity of subjects. Detailed procedures were disclosed to, and approved by human experimentation committees in each hospital studied. No subject was required to complete a questionnaire who did not wish to do so.

8. The research reported here was based at the University of Connecticut Health Center, Farmington, and is supported in parts by grants from the National Fund for Medical Education, the Commonwealth Fund, and the University of Connecticut Research Foundation.

9. A summary of this paper was presented at the Association of American Medical Colleges annual meeting, November, 1979.

10. Requests for reprints should be addressed to Dr. D. Candee, Department of Research in Health Education, University of Connecticut Health Center, Farmington, CT 06032.

11. Received for publication April 10, 1981.

12. Accepted for publication April 14, 1982. 